$10-2018$

\title{
Literary Tourism and La maison de Colette: An Interview with Frédéric Maget
}

Tama L. Engelking

Cleveland State University, t.engelking@csuohio.edu

Follow this and additional works at: https://engagedscholarship.csuohio.edu/clmlang_facpub

Part of the French and Francophone Language and Literature Commons

How does access to this work benefit you? Let us know!

\section{Publisher's Statement}

This manuscript published in The French Review. The full reference should be clearly indicated.

Please see citation below.

\section{Recommended Citation}

Engelking, Tama lea. "Literary Tourism and La maison de Colette: An Interview with Frédéric Maget."

French Review 92.1 (Oct. 2018): 184-97.

This Article is brought to you for free and open access by the Department of World Languages, Literatures, and Cultures at EngagedScholarship@CSU. It has been accepted for inclusion in World Languages, Literatures, and Cultures Faculty Publications by an authorized administrator of EngagedScholarship@CSU. For more information, please contact library.es@csuohio.edu. 


\title{
Literary Tourism and La maison de Colette: An Interview with Frédéric Maget
}

\author{
by Tama Lea Engelking
}

I France, where writers often have the status of rock stars, literary tourism is big business. As the most visited tourist destination in the world, with 84.5 million foreign visitors in 2015, France invests heavily in its patrimoine culturel by supporting the development of heritage sites: <entreprises.gouv.fr/files/files/ directions_services/etudes-et-statistiques/stats-tourisme/chiffres-cles/2016-keyfacts-tourism.pdf $>$. Among these are the sites associated with authors or books that comprise literary tourism. The growth of a more educated class of tourists, who are increasingly interested in using their leisure time to seek out cultural experiences, has contributed to the popularly of literary tourism sites such as les maisons d'écrivains - houses where writers were born, lived, or wrote, houses they frequently visited or those used as settings for their work (Mansfield 43). Although literary tourism dates back to classical antiquity, when it was common for travelers to make pilgrimages to the grave sites of great authors, the appeal of writers' houses is really a twentieth-century phenomenon. The 1980s and 1990s account for a quarter of the 201 maisons d'écrivains officially recognized by the Fédération nationale des maisons d'écrivains et des patrimoines littéraires.'

The house in the village of Saint-Sauveur-en-Puisaye, where Sidonie Gabrielle Colette was born and spent the first 18 years of her life (1873-1891), is one of the newest to join the Fédération. La maison de Colette, which first opened its doors to the public in 2016 and has since welcomed over 30,000 visitors, has also been recognized with the prestigious label of Maison des illustres. ${ }^{2}$ In the 2017 edition of Le guide vert Bourgogne, Michelin awarded a star to Colette's house, thus signaling that La maison de Colette is an "interesting" tourist destination according to nine 
different criteria that range from the first impression and the notoriety of the site to the quality of the visit and the reception given to visitors.

Although the fact that Colette grew up in a small village in Burgundy is an accident of history, the development of her house into an officially recognized monument historique was an act of tenacious commitment on the part of the Société des amis de Colette, which purchased the house in 2011. This is especially true of Frédéric Maget, the director of La maison de Colette and president of the Société. Maget was the driving force behind Colette en scène, a fund-raising gala held in Paris at the Théatre du Chatelet in 2010, which featured 18 artists who volunteered to perform texts by Colette. Among them were Leslie Caron, who played Gigi in the $1958 \mathrm{film}$ based on Colette's novella, the singer Juliette, and the actress Carole Bouquet. The success of that event, which raised enough money to secure the purchase of Colette's house, highlights the place that Colette still holds in the hearts of the French, as well as the important role that Maget, along with the association he directs, is playing to keep that interest alive and well.

As the first and only woman ever honored by the French government with a state funeral when she died in 1954, Colette enjoys an elevated status in le patrimoine littéraire. Maget's vision for La maison de Colette included restoring the house and gardens as authentically as possible in order to recreate the experience of the house as Colette would have known it when she lived there from 1873 to 1891 . This project also has a larger scope-to leverage Colette's reputation as a modern woman writer in order to serve as a bridge linking works from the past to works of the present. Centered around La maison de Colette, this effort aims to raise the status and visibility of women's participation in history and culture in the form of festivals, conferences, concerts, and exhibits, some of which have already taken place. ${ }^{3}$

The task of restoring La maison de Colette was a daunting undertaking for Maget and the team of restoration specialists he assembled, which included Pascal Prunet (architecte en chef des monuments historiques), Françoise Phiquepal (architectepaysagiste), and the decorator Jacques Grange. The restoration was a five-year $€ 1.5$ million project, which included 18 months of renovation characterized by an excruciating attention to detail in order to recreate the house as authentically as possible. This process, which is ongoing, includes locating and recovering some of the actual items owned by the Colette family-currently about $30 \%$ of the objects in the house-finding similar items, and recreating the lighting, wallpaper, flooring, blown glass window panes, hand-sewn curtains, garden design with the same configuration and variety of plants that Colette's mother Sido grew, and even the smells that would have permeated the rooms during Colette's childhood. A book specialist was charged with locating nineteenth-century editions of books that the family was known to possess, as well as journals to which Colette's father subscribed. Four hundred books are currently on display on the shelves lining 
the library, including Colette's personal set of Balzac's complete works with her extensive handwritten annotations."

Perhaps the biggest challenge facing Maget before opening the doors of Colette's house was deciding how to present the house to visitors--how to stage the actual guided tour of the rooms and garden. As David Herbert has noted, literary places are "social constructions, created, amplified, and promoted to attract visitors" who come to these sites for a variety of reasons and with many different expectations for what they will experience there (313). How will the material reality of walking in Colette's footsteps, of seeing the same view of the village she saw from her bedroom window as a little girl, affect the literary tourist? What new understanding of Colette will visitors to her house have when they leave? And when they exit through the gift shop, will they leave with a book by Colette in hand? Although studies show that many visitors to writers' houses are enthusiastic readers who are drawn to places associated with the writers they admire, tourists often visit literary places "for some broader and deeper emotion than the specific writer or the story" (Herbert 314). Statistics indicate that as many as one-third of the visitors to a writer's house may have never read a word of the author's works: <litteraturelieux.com/multimedia/File/publications/2008-etude-publics-lieux-litteraires.pdf $>$. Some may have seen a reference in a guide book or online, and others may be visiting the region for other reasons and decide to tour a writer's house based on recommendations by friends or the proximity to other places on their itinerary. Whatever the case, the managers of these sites have an opportunity to create a specific image of the writer by choosing to highlight certain aspects of the writer's life and work and connecting these to the physical space and objects in the house the writer once inhabited.

To create the narrative framework of the guided visits to La maison de Colette, Maget reread all of Colette's works and let himself be guided by the words of Colette, who was, above all, a storyteller. Encouraged by her stepson Bertrand de Jouvenel, with whom she had visited the house in Saint-Sauveur in 1920, Colette wrote down the stories she was fond of telling about her childhood. The result was La maison de Claudine (1922), a collection of freestanding anecdotes about the house, garden, village, and family members, which Anne Freadman calls the first of Colette's livres-souvenirs (58). Freadman describes the vignettes that make up the chapters of this book as "scattered fragments of memories" that Colette assembled to tell stories about her past. Maget has reassembled many of these fragments and others gleaned from Colette's writings to make La maison de Colette come alive for visitors. Maget and his team of professional guides draw on Colette's storytelling legacy by integrating quotes from Colette into every aspect of the visit. They've staged Colette's house as a sort of "maison-livre" with the experience of moving from room to room comparable to turning pages in a book. 
In Writers' Houses and the Making of Memory, Harald Hendrix reminds us that writers' houses are "highly susceptible to manipulation" by both the writer and the manager responsible for transforming a private space into a public space (3). As an expressive medium, a writer's house can be used by the author who lived there as a means of self-fashioning, which Colette certainly did. ${ }^{5}$ Although she never wrote during the years she spent in Saint-Sauveur, and, to the contrary, insisted quite emphatically that during her childhood she felt she was precisely born not to write, numerous scholars have linked the origins of Colette's vocation to the sensibilities she developed in the rich sensory world of her childhood. ${ }^{6}$ By titling her collection of childhood reminiscences La maison de Claudine, Colette created an explicit link between her natal home and her first published work, Claudine à l'école (1900), which she set in a thinly disguised version of her hometown. As Joan Hinde Stewart has remarked, the past that Colette treats in $\mathrm{La}$ maison de Claudine is a literary past, and its very title "places it under the sign of the author's entrance into literature" (266).

Visitors to La maison de Colette come to Saint-Sauveur-en-Puisaye with many different expectations and motivations. They may or may not be seeking insights into Colette's work and her origins as a writer, but they are all seeking an experience that is unique to this specific place. One quality that makes writers' houses so alluring is what Hendrix calls the "through-the-looking-glass" effect that allows visitors, through contact with physical space and objects, to fuse the real and imaginary, fact and fiction, and to "come into contact with the imaginative world created by the author, and thus to participate in his imagination" (239). Specialists in literary tourism identify the tripartite "auteur-œuvre-lieux" as an evocative combination that creates a resonance between the imaginary and an individual's memories and perceptions when they visit a writer's house: " $\AA$ travers cette expérience, le visiteur relie l'œuvre au lieu et relit l'œuvre, le lieu ajoute du sens et même un double sens, à la fois signifiant et sensoriel" (Bonniot-Mirlou and Blasquiet 16). In her study of the nineteenth-century bourgeois interiors of writer's houses, Diana Fuss finds a relationship between interior spaces and the subjective identities that writers create "through a sensory identification with things" (15). Sensory knowledge, she suggests, serves as a bridge between the architectural and the psychological, which explains the impact that physically experiencing a writer's house can have on literary tourists when an author, her house, and her works resonate together, as is the case with Colette. The authenticity of the spaces and objects staged in a writer's house can heighten this impact. The concept of authenticity in tourism studies recognizes the special nature of original objects, which have a special 'aura' that is lost by mass production (Fawcett and Cormack 700 ). Authenticity is also viewed as a subjective experience, "a combination of the developers' intentions, the consumers' interpretations, and the interactions among 
them" (Herbert 317). Whatever the intended message, tourists are active consumers who, much like readers, interpret their personal experiences in diverse ways.

La maison de Colette seems specifically staged to enhance the tripartite combination and the authentic aspects of the site, an observation reinforced by my own experience there. I had the pleasure of visiting La maison de Colette on a sunny spring day in 2017, where I was treated to a guided tour by Maget. Our group included a dozen middle-school students who had never read Colette but were assigned to find a personal connection to the author and write about it. During my stay in Saint-Sauveur, I also toured Colette's house with other groups that ranged from members of a local hiking club to middle-aged couples, families, and older women who, like me, seemed to know Colette's work well. According to Maget, the average age of visitors to La maison de Colette is 50-55. Although French visitors in this demographic are likely to have read Colette in school, her works are no longer required reading. Maget hopes to change this through outreach efforts to local schools and by developing pedagogical materials for students who visit La maison de Colette.

I interviewed Maget in Saint-Sauveur-en-Puisaye in April 2017 and asked him about his vision for La maison de Colette, its unique features, and what a visit to Colette's house can offer to her readers. If my own experience is any indication, the personal connection I made with Colette's world during my visits to La maison de Colette and the village of Saint-Sauveur-en-Puisaye has convinced me of the value of literary tourism, and especially how physically experiencing a writer's house can provide insights and enhance our understanding of an author and her works.

Q: Frédéric Maget, professeur de lettres modernes, président de la Société des amis de Colette, directeur de La maison de Colette, auteur et rédacteur de plusieurs œuvres sur Colette, notamment Cahier Colette (L'Herne, 2011), Colette journaliste (2014), Colette: lettres à Missy (2009), vous avez consacré une grande partie de votre vie professionnelle à Colette. On peut parler d'une vocation ou même d'une passion de faire redécouvrir la personne et l'œuvre de Colette. D'où vient cette passion?

R: Comme toutes les véritables passions, ma passion pour Colette a des origines multiples et sans doute, pour partie, inconscientes. J'ai découvert Colette à l'âge de 13 ou 14 ans. J'étais alors au collège et je passais beaucoup de temps à la bibliothèque de ma commune. Au hasard de mes lectures, je suis tombé sur La vagabonde. Mon choix n'était motivé par aucune raison intellectuelle ou littéraire puisque j'appartiens à une génération qui n'a pas étudié Colette à l'école. Ma famille, plus scientifique que littéraire, ne m'avait elle-même transmis aucune représentation la concernant. L'enchantement, le charme-au sens étymologique du terme-agit puissamment. Je ne saurai démêler, même aujourd'hui, la somme 
d'impressions qui accompagnèrent ma lecture. La langue, le style, le personnage de Renée Néré, sa solitude, son stoïcisme, une forme de sagesse dans la façon de parler des êtres et de l'amour, la langue, le choix des mots. Tout cela m'a immédiatement séduit. Ce fut le début d'un compagnonnage qui dure depuis bientôt trente ans. La passion, bien sûr, doit être entretenue et s'enrichir de rencontres. J'ai eu la chance de croiser sur ma route des passeurs, comme Alain Brunet, Marguerite Boivin, Michel Remy-Bieth ou Gérard Bonal, qui m'ont encouragé et m'ont donné envie d'approfondir mes connaissances. Petit à petit le simple lecteur s'est transformé en commentateur et en éditeur, en secrétaire général puis en président des amis de Colette, aujourd'hui en directeur de la maison natale. C'est finalement un parcours assez linéaire, cohérent et simple.

Q: Dans votre rôle comme président de la Société des amis de Colette et directeur de La maison de Colette (un fonds de dotation) à laquelle la maison natale de Colette appartient, vous devez porter de nombreuses casquettes-publicitaire, homme d'affaires et politique qui maintient des liens avec des associations régionales et nationales (la mairie de Saint-Sauveur-en-Puisaye, le Conseil départemental de l'Yonne, le Conseil régional de Bourgogne-Franche-Comté, le ministère de la Culture et de la Communication, des institutions telles que la Fédération des maisons d'écrivains, etc.), gérant d'un projet culturel qui comprend La maison de Colette ainsi que les activités académiques, touristiques, pédagogiques associées (Festival des écrits de femmes, conférences, spectacles, visites scolaires, etc.). J'ai l'impression que toutes ces activités sont assez loin de votre formation universitaire en lettres modernes, mème si votre connaissance et appréciation des cuvres de Colette sont à la base de tout ce que vous faites. Parlez-moi de vos rôles divers, des défis et des plaisirs que ces rôles vous ont apportés, et de ce qu'on appelle en anglais votre "learning curve" (courbe d'apprentissage) en ce qui concerne La maison de Colette.

R: J'ai, en effet, beaucoup de "casquettes", comme on dit. J'exerce des tâches diverses qui vont de l'événementiel à la gestion des stocks d'une librairie, en passant par les ressources humaines, les relations avec les collectivités, les mécènes, les éditeurs, la presse. Des activités qui sont à mille lieux de ce que j'ai appris en classes préparatoires ou à l'université. J'ai heureusement la chance d'être entouré de gens de grande qualité qui me font partager leur expérience. Nous sommes une toute petite équipe. Nous fonctionnons sans aides publiques. La polyvalence est donc une nécessité. Pourtant, j'ai le sentiment qu'il y a, au fond, une continuité avec mon ancien métier d'enseignant-je suis d'ailleurs toujours officiellement fonctionnaire de l'Éducation nationale. Dans tout ce que je fais, il y a toujours le même souci du partage et de la transmission. Mon expérience auprès des élèves et des étudiants, dans des milieux très différents, m'a été très utile pour concevoir la visite de la 
maison et même, au moment des travaux de réhabilitation, dans le choix des meubles et des objets, car j'avais constamment en tête ce que les guides pourraient en faire et ce qu'ils pourraient apporter aux visiteurs. La maison de Colette est, comme le dit Julia Kristeva, une "initiation à la lecture" de l'œuvre, et mon plus grand plaisir est de voir les visiteurs acheter les ouvrages de Colette en quittant la maison.

Q: Vous avez indiqué que Nohant (la maison de George Sand), Monk's House (la maison de Virginia Woolf) et Vulaines (la maison de Stéphane Mallarmé) ont servi de modèles pour La maison de Colette. Qu'est-ce qu'elles ont en commun, et qu'est-ce qui distingue la maison de Colette des autres maisons d'écrivains?

R: Le premier point commun est sans doute l'importance de ces maisons dans la vie et l'œuvre des trois écrivains. Le second est le parti pris muséographique de chacun des lieux. À Monk's House comme à Nohant, comme à la maison de Colette aujourd'hui, on rentre dans chaque maison comme si les occupants venaient de s'absenter. Pas de fils, de cordons ni de barrières, on circule à travers les pièces au plus près des meubles et objets qui ont constitué le quotidien de chacune des créatrices. À Vulaines, chez Mallarmé, l'intervention muséographique a été plus poussée (il y a des fils et des aménagements contemporains pour l'éclairage), mais j'y ai retrouvé le même souci de restituer au plus près l'intimité du lieu. Autre point commun, l'importance des jardins et, au-delà, du cadre et de l'environnement, qui jouent beaucoup dans l'appréciation que l'on peut avoir du lieu. Le parc et le petit village de Nohant, les jardins fleuris avec l'ouverture sur le village dans le cas de Monk's House, le verger et la proximité de la Seine dans le cas de Mallarmé. Tout cela se retrouve chez Colette à un degré à mon sens-mais je ne suis pas objectifsupérieur encore: importance cruciale des jardins, ouverture sur le village et la campagne environnante devenus eux-mêmes sources d'inspiration pour l'écrivaine. Du point de vue de la médiation, la maison de Colette se rapproche de Nohant, puisque comme chez George Sand on ne visite la maison de Colette que par le biais d'une visite guidée qui permet d'accompagner la découverte des lieux de récits et d'anecdotes. Dans le cas de Monk's house, qui reste pour moi la réussite absolue, il n'y avait—du moins lorsque je l'ai visitée-pas de guide mais, dans chaque pièce, réparties discrètement, des petites notices éclairant tel ou tel détail du décor. Ả Vulaines, on peut également visiter librement les espaces avec un guide imprimé ou quelques notices plus "professionnelles" réparties tout au long du parcours de visite.

En évoquant pour vous ces trois lieux fondateurs dans mon imaginaire de "la maison d'écrivain", je me dis également que leur point commun-avec leurs différences que je viens de signaler-c'est que chacun propose une expérience de visite où la sensibilité et l'émotion jouent un rôle essentiel. C'est bien cela que nous essayons de proposer à la maison de Colette: "une expérience" de visite, un moment 
privilégié, un peu hors du temps, en compagnie des textes de Colette. Mais il me semble néanmoins que la maison de Colette se différencie des trois autres lieux sur un point fondamental. Nohant, Monk's House, Vulaines ont été des lieux de création et, en tout cas pour Monk's House et Vulaines, pensés comme tels, éloignés de la ville, de la foule et du bruit, conçus pour offrir à l'écrivaine ou à l'écrivain le cadre idéal favorable à son travail. Nohant est de ce point de vue plus proche de la maison de Colette, car il s'agit d'une maison liée à l'histoire familiale, mais Nohant fut pour Sand un lieu de sociabilité littéraire et un lieu d'écriture. Or Colette n'a pas écrit dans sa maison. Abandonnée alors que Colette avait dix-huit ans, récupérée quand elle en avait cinquante sans qu'elle puisse s'y installer de nouveau, la maison (et ses jardins) fut à jamais pour Colette un Eden perdu, un paradis qu'il fallait reconquérir par l'écriture et l'imagination. La maison n'est pas le lieu de la création: elle est la création, un véritable personnage dans l'œuvre et mème un rouage de l'écriture. "Une maison-livre" pour reprendre l'expression de Jéròme Garcin (20). En cela la maison de Colette est unique et ne peut se comparer qu'à Illiers-Combray pour Marcel Proust, sauf que le jeune Marcel n'y a peut-être passé en tout et pour tout que deux mois et que malgré l'importance fondatrice du lieu, il n'innerve pas, au point où c'est le cas pour Colette, toute une œuvre.'

Q: Pour reprendre les mots de Kristeva, sauver la maison natale de Colette "participe non pas d'un culte, mais d'une initiation à la lecture de son œuvre" (36). Quel est le lien entre la maison de Colette et ses œuvres? Quel rôle ont-elles joué dans la reconstitution de sa maison et la visite guidée que vous avez mise en place pour les visiteurs? Pour un visiteur "typique" à la maison de Colette, quelle valeur cette expérience est-elle censée contribuer au lecteur de ses œuvres?

R: J'ai retrouvé un écho récent de cette affirmation de Kristeva dans un entretien avec Jean-Michel Leniaud: "La maison de Colette, à Saint-Sauveur-en-Puisaye [...] mérite d'être sauvegardée si l'on veut comprendre son œuvre" (8). Le lien entre la maison de Colette et l'œuvre de Colette est consubstantiel. On peut légitiment estimer que Colette n'aurait pas écrit Claudine à l'école, son premier roman, paru en 1900, si elle n'avait pas ressenti le besoin-la nécessité-de réparer littérairement le traumatisme du départ de Saint-Sauveur à l'automne 1891. C'est encore vers la maison qu'elle se tourne à l'approche de la cinquantaine pour renouveler son œuvre, alors principalement consacrée, en dehors des Claudine, aux cocottes et aux artistes du music-hall. La maison de Claudine, parue en 1922, va initier un nouveau cycle dans la création: l'évocation du monde de l'enfance, et bien sûr le personnage de Sido qui fait sa première apparition sous son nom dans ce recueil et qui va devenir, pour reprendre les mots de Colette, "le personnage principal de toute ma vie". Des chefs-d'œuvre comme Sido (1930) ou La naissance du jour (1928) doivent tout à ce retour vers la maison natale. Ce n'est d'ailleurs pas un hasard si la plupart des héroïnes de Colette, jusqu’à Julie de Carneilhan, dans le 
roman éponyme, en 1941, prennent également "le chemin du retour". Au total, ce sont des dizaines de pages réparties dans l'œuvre pendant près d'un demi-siècle depuis le premier roman jusqu'au dernier texte publié, Ces dames anciennes (1954). Je ne connais d'autre exemple dans l'histoire de la littérature française d'un auteur qui ait consacré autant de pages, autant d'énergie créatrice, à recomposer un lieu aussi étroitement lié à la vie du créateur. Le plus étonnant pour nous, lorsque nous avons entrepris la restauration de la maison, fut de constater l'extrême précision de ces souvenirs. Colette nous avait offert une sorte de mode d'emploi décrivant le motif et la couleur des papiers peints et des tentures, l'ameublement des pièces, depuis la matière et les motifs.

Q: Dans l'introduction au numéro du Cahier de l'Herne que vous avez rédigé sur Colette avec Gérard Bonal, vous présentez Colette comme une femme moderne et subversive. Pour certains, Colette représente le modèle d'une femme libre, dont le comportement, parfois scandaleux, souvent révolutionnaire, exprime sa volonté de faire ce qu'elle voulait tout au long de sa vie. Selon ses propres mots, qui servent de sous-titre à la biographie écrite par Gérard Bonal: "Je veux faire ce que je veux". Cependant, d'autres l'identifient avec ses racines bourguignonnes et son rapport avec la nature et les animaux. Ces images de Colette sont-elles en conflit? La maison de Colette, qu'est-ce qu'elle contribue à notre compréhension du trajet qui mène de la jeune fille poyaudine à la grande écrivaine Colette?

R: Je crois, tout d'abord, qu'il ne faut pas nécessairement chercher à résoudre les contradictions et les paradoxes d'un être ou d'une œuvre. Une des richesses de Colette et ce qui fait, peut-être, que sa vie et son œuvre résistent au temps, c'est qu'elle n'est pas une, mais multiple. Toutes les images de Colette-la nature, les chats, les scandales, la liberté, le féminisme avant l'heure, etc.-sont exactes et partielles à la fois. Michèle Sarde avait sous-titré sa biographie "Libre et entravée", soulignant ainsi les contradictions qui sont au cour de l'œuvre. Bourgeoise par ses aspirations au confort et à la reconnaissance sociale, Colette est aussi provocatrice, se moque des conventions de la bien-pensance, chante les mérites et la poésie du petit-monde des théâtres et de la rue. Elle incarne à la fois la Parisienne et la Bourguignonne, attachée à sa terre natale, faisant sonner son accent rocailleux jusque dans ses dernières années.

En parcourant la maison, le visiteur découvre au fur et à mesure des pièces et des anecdotes la vie de Gabrielle Colette et de sa famille, la personnalité atypique de ses parents, Sido et le Capitaine, l'hostilité du village, le contact privilégié avec la nature, notamment dans les jardins. Tout est déjà là: un décor bourgeois de province menacé par la ruine, la haine des habitants de Saint-Sauveur à l'égard de la mécréante Sido, "l'étrangère" dans le village, la conduite provocatrice de Sido, notamment à l'église où elle emmène son chien aboyer à la messe, l'attention de Sido aux plus humbles, aux filles-mères, l'éducation de Sido qui apprend à ses 
enfants la valeur de la différence ("ne pas chercher à ressembler aux autres"), la bibliothèque où Colette pourra puiser très librement, sa découverte de Balzac, dont on n'a pas encore assez dit l'influence, les "pages blanches" du Capitaine qui sont pour partie dans la vocation littéraire de sa fille, et puis les jardins, bien sûr, où Colette apprend au contact de Sido l'art du regard, le respect de la vie animale, l'attention aux éclosions. Tout est là, je le répète. À l'état brut. La vie et le difficile métier d'écrire feront de tout ce matériau une œuvre qui compte parmi les plus évocatrices et les plus singulières de notre littérature.

Q: L'ouverture de la maison de Colette au public n'est que la première étape d'une vision plus large. Quels sont les projets futurs associés à la maison de Colette?

R: J'ai souhaité que la maison de Colette, conformément à ce que je viens de dire, ne fixe pas une image unique de Colette, celle du paradis de l'enfance et du bonheur provincial. C'est pourquoi j'ai développé, en plus du projet patrimonial, un projet culturel dont l'objectif est de confronter l'œuvre de Colette à celle d'autres créateurs, et notamment d'autres créatrices, du passé et du présent. Des conférences, des projections, des festivals sont organisées tout au long de l'année, dans la maison ou dans d'autres lieux du village, qui partent de l'univers de Colette et ouvrent sur d'autres univers, comme le festival "Comme ça me chante!" qui met en valeur le goút de la musique et l'écriture musicale de Colette en faisant se rencontrer sur scène musiciens et comédiens, ou bien encore le Festival international des écrits de femmes, qui propose de redécouvrir les grandes figures féminines de notre histoire.

La maison elle-même deviendra, dans les mois ou les années qui viennent, un lieu de transmission, par l'accueil du public scolaire et l'organisation des ateliers culinaires pour les enfants dans la cuisine de Sido, et avec l'aménagement, dans les combles de la maison, d'un lieu de conservation et de consultation d'archives concernant Colette, associés à un studio pour accueillir un auteur ou un chercheur en résidence. Le projet pourrait également, dans les années qui viennent, fédérer tout un village. Nous avons déjà créé, dans Saint-Sauveur, un parcours "Sur les pas de Colette", qui permet de découvrir derrière une trentaine de façades les personnages et les anecdotes racontées par Colette dans son ouvre. Nous travaillons à repenser avec les élèves du collège "le sentier Colette", qui part dans la campagne environnante à la découverte des paysages décrits dans l'œuvre. Nous essayons de persuader la commune de la nécessité d'un fonctionnement commun et intégré avec le musée Colette qui se trouve dans le château, un lieu complémentaire puisqu'on peut, notamment, y découvrir une partie du mobilier du Palais-Royal, la dernière demeure de l'écrivain. Saint-Sauveur deviendrait alors un village littéraire offrant la possibilité de passer tout un week-end sur les pas de Colette. ${ }^{8}$ 


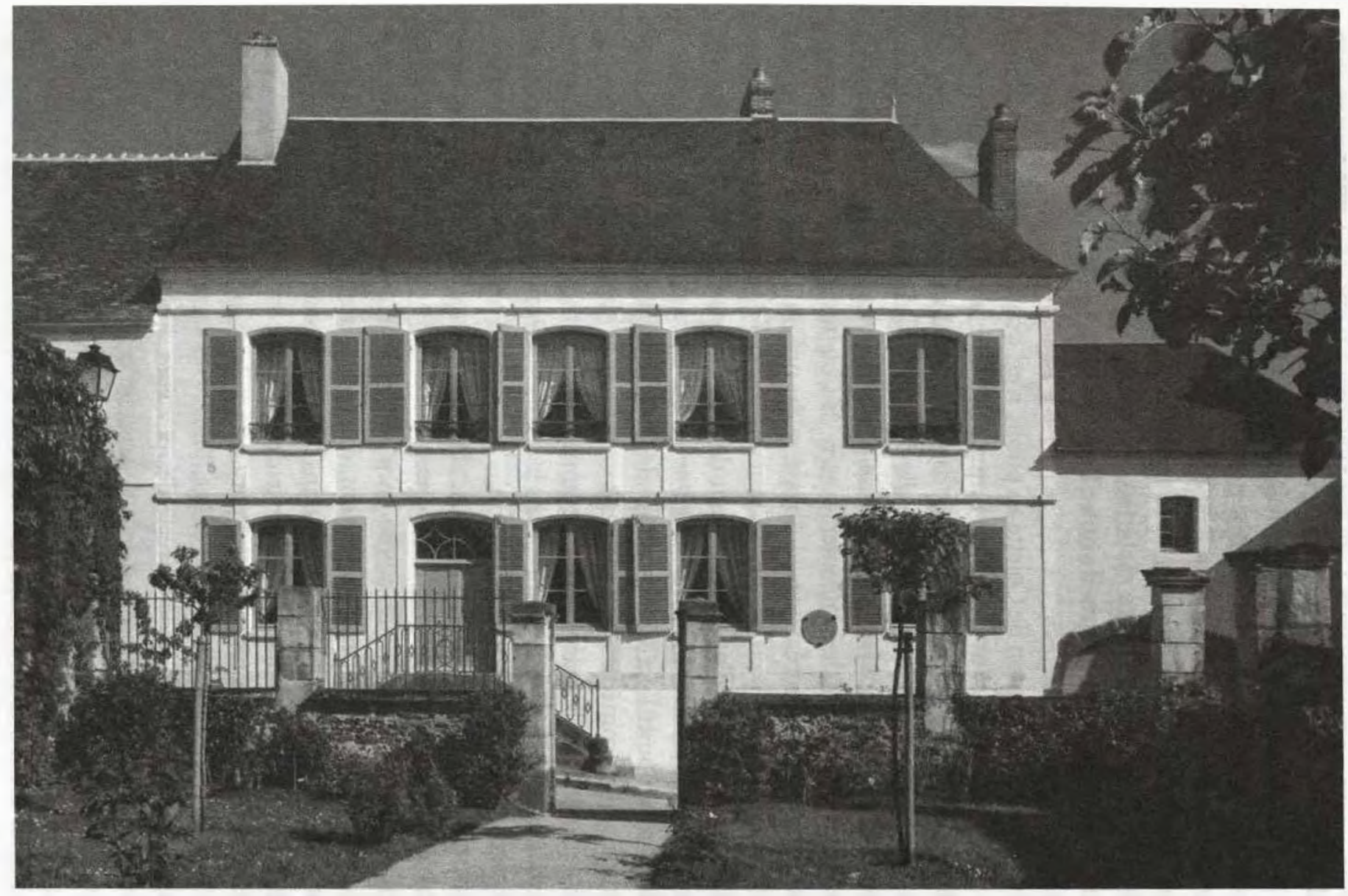

Restored façade of La maison de Colette. Used with permission from La maison de Colette and Nicolas Castets. 


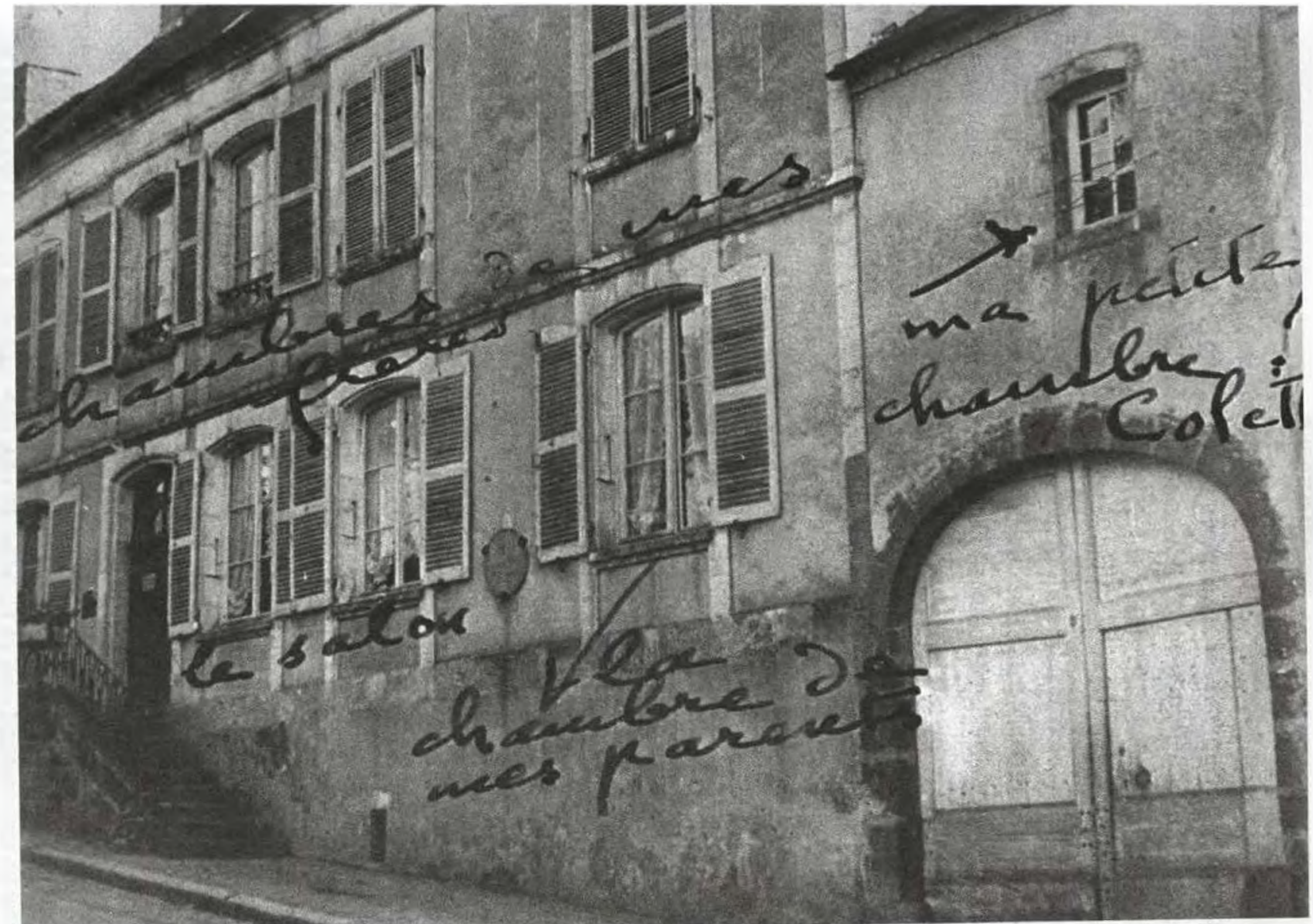

Postcard with Colette's handwritten labels identifying the rooms in her home. Used with permission from La maison de Colette. 


\section{Notes}

'France is particularly rich in maisons d'écrivains, which draw over one million visitors each year. Some of the best known include Victor Hugo's house on Place des Vosges in Paris and George Sand's house in Nohant. For an interactive map of les maisons d'écrivains, see <litterature-lieux.com>. Only certain writers' houses meet the criteria to qualify for the more prestigious label of Maison des illustres.

${ }^{2}$ Maison des illustres is a label created by the ministere de la Culture in 2011 in order to draw public attention to "les lieux dont la vocation est de conserver et transmettre la mémoire de femmes et d'hommes qui les ont habitées et se sont illustrés dans l'histoire politique, sociale et culturelle de la France." There are currently only 212 houses with this label: <culturecommunication.gouv.fr/Aides-demarches/Pro tections-labels-et-appellations/Label-Maisons-des-Illustres $>$.

"'Le Festival international des écrits de femmes" (FIEF), which debuted at La maison de Colette in 2012, is part of that effort. The 2016 festival, organized around the theme of "Féminismes," included a roundtable discussion of Colette's feminism and talks by writers such as Julia Kristeva, who devoted the third volume of Le génie féminin to Colette. For La maison de Colette and related activities, see <maison decolette.fr>.

${ }^{4}$ For photos and details about the process of rehabilitating the house and gardens, see the special issue of Connaissance des arts devoted to La maison de Colette.

${ }^{5}$ Proust's apartment on the Boulevard Haussmann is a much-studied example (Kear; Fuss).

'Delacour-Hennart considers La maison de Claudine as "l'œuvre fondatrice de la vocation de Colette" (95). Other scholars who develop this connection include Freadman, Hinde Stewart, and Schechner.

'Maget's comparison of Colette's house with Nohant has another dimension. Although Nohant rates among the ten most visited sites in France, the guided tour of the house concentrates primarily on the life of George Sand. Consequently, as BonniotMirloup and Blasquiet point out, a visit to Nohant "n'évoque l'œuvre qu'à travers son contexte d'écriture. Il faut s'éloigner de la maison pour trouver des références à l'imaginaire des cuvres un peu partout sur le territoire" (15). A personal anecdote bears out this difference with Colette's house. I visited La maison de Colette in the company of a friend who had never read Sand or Colette. Following our tour, she asked me to recommend one of Colette's books. She admitted that although she has visited Nohant three times, she has never felt moved to read any of Sand's works.

${ }^{8}$ I would like to thank the staff of La maison de Colette, Samia Bordji, head of the Centre d'études de Colette, and especially Frédéric Maget, directeur de La maison de Colette, for their generosity and patience in answering all my questions about the house and guided tour. This research was made possible by funding from a Faculty Scholarship Initiative grant provided by Cleveland State University. 


\section{Works Cited}

Bonal, Gérard. Colette: "Je veux faire ce que je veux". Perrin, 2014.

Bonal, Gérard, et Frédéric Maget, éd. Colette. L'Herne, 2001.

. Colette journaliste: chroniques et reportages, 1893-1955. Seuil, 2010.

Bonniot-Mirloup, Aurore, et Hélène Blasquiet. "De l'œuvre aux lieux: la maison d'écrivain pour passerelle (France)". Territoire en mouvement. Revue de géographie et d'aménagement 31 (2016): 1-19. <tem.revues.org/3722>.

Colette. CEuvres. Ed. Claude Pichois et al. 4 vols. Gallimard 1984-2001.

Connaissance des arts hors-série. La maison de Colette 789 (septembre 2017).

Delacour-Hennart, Audrey. "De Claudine à l'école à La maison de Claudine: aux origines de la vocation de Colette". Roman 20-50 6 (2016): 87-100.

Fawcett, Claire, and Patricia Cormack. "Guarding Authenticity at Literary Tourism Sites." Annals of Tourism Research 28.3 (2001): 686-704.

Freadman, Anne. The Livres-Souvenirs of Colette: Genre and the Telling of Time. Legenda, 2012.

Fuss, Diana. The Sense of an Interior: Four Writers and the Rooms that Shaped Them. Routledge, 2004.

Garcin, Jérôme. "La maison-livre de Colette". Programme pour Colette en scène au Théatre du Châtelet, Paris, Playbill, 2010. 20-21.

Hendrix, Harald, ed. Writers' Houses and the Making of Memory. Routledge, 2012. . "Epilogue: The Appeal of Writers' Houses." Hendrix 235-43.

"Writers' Houses as Media of Expression and Remembrance: From Self-

Fashioning to Cultural Memory." Hendrix 1-12.

Herbert, David. "Literary Places, Tourism and the Heritage Experience." Annals of Tourism Research 28.2 (2001): 312-33.

Leniaud, Jean-Michel. "On peut aimer le patrimoine sans être réactionnaire". Entretien.

L'express théma: trésors cachés de France 4 juillet 2017: 6-11.

Kear, Jon. "'Une chambre mentale': Proust's Solitude." Writers' Houses and the Making of Memory. Ed. Harald Hendrix. Routledge, 2012. 221-34.

Kristeva, Julia. "Colette, ou la chair du monde". Programme pour Colette en scène au Théatre du Châtelet, Paris, Playbill, 2010. 36-37.

. Le génie féminin: les mots-Colette ou la chair du monde. Vol. 3. Fayard, 2002.

Maget, Frédéric, et Samia Bordji, éd. Colette: lettres à Missy. Flammarion, 2009.

Mansfield, Charlie. Researching Literary Tourism. Shadows, 2015.

Sarde, Michèle. Colette, libre et entravee. Stock, 1991.

Schechner, Stephanie. "A Delicate Balance: Becoming a Woman and a Writer in

Colette's Claudine d l'école and La maison de Claudine." Dalhousie French Studies

67 (Summer 2004): 75-87.

Stewart, Joan Hinde. "The School and the House." Women's Studies 8.3 (1981): 25972. 